\title{
Detection of the KIT D816V mutation in peripheral blood of systemic mastocytosis: diagnostic implications
}

Maria Jara-Acevedo ${ }^{1}$, Cristina Teodosio ${ }^{2}$, Laura Sanchez-Muñoz ${ }^{3}$, Ivan Álvarez-Twose ${ }^{3}$, Andrea Mayado ${ }^{1}$, Carolina Caldas ${ }^{1}$, Almudena Matito ${ }^{3}$, José M Morgado ${ }^{3}$, Javier I Muñoz-González ${ }^{1}$, Luis Escribano ${ }^{1}$, Andrés C Garcia-Montero ${ }^{1,4}$ and Alberto Orfao ${ }^{1,4}$

${ }^{1}$ Servicio General de Citometría (NUCLEUS), Centro de Investigación del Cáncer (IBMCC-CSIC/USAL and IBSAL) and Departamento de Medicina, Universidad de Salamanca, Salamanca, Spain; ${ }^{2}$ Department of Immunology, Erasmus MC, University Medical Center Rotterdam, Rotterdam, The Netherlands and ${ }^{3}$ Centro de Estudios de Mastocitosis de Castilla La Mancha (CLMast), Hospital Virgen del Valle, Toledo, Spain

\begin{abstract}
Recent studies have found the KITD816V mutation in peripheral blood of virtually all adult systemic mastocytosis patients once highly sensitive PCR techniques were used; thus, detection of the KIT D816V mutation in peripheral blood has been proposed to be included in the diagnostic work-up of systemic mastocytosis algorithms. However, the precise frequency of the mutation, the biological significance of peripheral blood-mutated cells and their potential association with involvement of bone marrow hematopoietic cells other than mast cells still remain to be investigated. Here, we determined the frequency of peripheral blood involvement by the KIT D816V mutation, as assessed by two highly sensitive PCR methods, and investigated its relationship with multilineage involvement of bone marrow hematopoiesis. Overall, our results confirmed the presence of the KIT D816V mutation in peripheral blood of most systemic mastocytosis cases $(161 / 190 ; 85 \%)$-with an increasing frequency from indolent systemic mastocytosis without skin lesions $(29 / 44 ; 66 \%)$ to indolent systemic mastocytosis with skin involvement (124/135; 92\%), and more aggressive disease subtypes $(11 / 11 ; 100 \%)$-as assessed by the allele-specific oligonucleotide-qPCR method, which was more sensitive $(P<.0001)$ than the peptide nucleic acid-mediated PCR approach $(84 / 190 ; 44 \%)$. Although the presence of the $K I T$ mutation in peripheral blood, as assessed by the allele-specific oligonucleotide-qPCR technique, did not accurately predict for multilineage bone marrow involvement of hematopoiesis, the allele-specific oligonucleotide-qPCR allele burden and the peptide nucleic acid-mediated-PCR approach did. These results suggest that both methods provide clinically useful and complementary information through the identification and/or quantification of the KIT D816V mutation in peripheral blood of patients suspected of systemic mastocytosis.
\end{abstract}

Modern Pathology (2015) 28, 1138-1149; doi:10.1038/modpathol.2015.72; published online 12 June 2015

Systemic mastocytosis is a group of heterogeneous disorders characterized by an abnormal accumulation of clonal mast cells in different tissues, such as the bone marrow, skin, spleen and/or the gastrointestinal tract. ${ }^{1}$ Owing to the heterogeneity of the disease, the current WHO 2008 classification of

Correspondence: Dr AC Garcia-Montero, PhD or Professor A Orfao, MD, PhD, Centro de Investigación del Cáncer (IBMCC-CSIC/USAL and IBSAL) and Departamento de Medicina, Universidad de Salamanca, Campus Miguel de Unamuno, Salamanca 37007, Spain.

E-mail: angarmon@usal.es or orfao@usal.es

${ }^{4}$ These authors contributed equally to this work.

Received 9 March 2015; accepted 5 May 2015; published online 12 June 2015 mastocytosis ${ }^{2}$ defines seven different diagnostic categories of the disease, which vary from clinically indolent to aggressive forms of systemic mastocytosis. From all WHO subtypes of mastocytosis, indolent systemic mastocytosis is by far the most prevalent one in adults. ${ }^{3}$ At diagnosis, the great majority of adult indolent systemic mastocytosis cases present with both skin and bone marrow involvement, although a significant number of indolent systemic mastocytosis cases also show symptoms associated with the release of mast cells mediators in the absence of skin lesions at diagnosis. ${ }^{4,5}$ Interestingly, virtually all $(>90 \%)^{6}$ indolent systemic mastocytosis and aggressive systemic mastocytosis patients carry the KIT D816V 
somatic mutation in pathological mast cells from the skin and bone marrow, the latter contributing (as a minor criteria) to the diagnosis of systemic disease. As the level of infiltration of bone marrow by tumor mast cells is frequently $\operatorname{low}^{7}$ (eg $<0.1 \%$ of bone marrow-aspirated samples), highly sensitive molecular approaches have long been required for reliable detection of the KIT D816V mutation, even after purification of bone marrow mast cells. ${ }^{8,9}$ Despite this, it is now well established that, in at least onethird of indolent systemic mastocytosis and all aggressive systemic mastocytosis patients, bone marrow myeloid and/or lymphoid cells other than mast cells also carry the KIT D816V mutation (multilineage systemic mastocytosis); ${ }^{6}$ such patients have a greater risk for disease progression. ${ }^{10}$ Among multilineage systemic mastocytosis patients, the presence of the KIT D816V mutation is typically detected in genomic DNA (gDNA) of CD34+ hematopoietic stem and precursor cells, eosinophils, monocytes and maturing neutrophils, and, to a less extent, also in $\mathrm{T}$ lymphocytes, in addition to bone marrow mast cells. ${ }^{6,11}$

Altogether, these results indicate that clonal mast cells from adult indolent systemic mastocytosis and aggressive systemic mastocytosis patients derive from a hematopoietic precursor with the ability for multilineage differentiation, which already carries the KIT D816V mutation. ${ }^{6,12}$ Thereby, the KIT mutation should also be detectable in peripheral blood from a significant percentage of systemic mastocytosis cases. ${ }^{10}$ In line with this hypothesis, new highly sensitive methods have been described, ${ }^{13}$ which allowed detection of the KIT D816V mutation in peripheral blood leukocytes from most mastocytosis cases, including 66/69 (96\%) indolent systemic mastocytosis and $3 / 4$ cutaneous mastocytosis investigated. ${ }^{9,14}$ Despite this, the potential relationship between the presence of the KIT D816V mutation in peripheral blood and multilineage involvement of bone marrow hematopoiesis has not been investigated so far. In this regard, particularly intriguing is the potential lack of association between the allele burden for the KIT mutation in peripheral blood, as assessed by the allele-specific oligonucleotide polymerase chain reaction (allelespecific oligonucleotide-qPCR), and the clinical and biological features of indolent systemic mastocytosis. ${ }^{9,15}$ Thereby, the precise biological and clinical significance of the presence of circulating peripheral blood KIT-mutated cells in systemic mastocytosis still remains to be elucidated.

Here, we investigated the presence of the KIT D816V mutation in peripheral blood from a series of 190 KIT $\mathrm{D} 16 \mathrm{~V}^{+}$systemic mastocytosis and 2 KIT D816V ${ }^{+}$monoclonal mast cell activation syndrome ${ }^{5,16}$ cases using a peptide nucleic acid-mediated PCR-clamping (peptide nucleic acidmediated PCR) technique ${ }^{17}$ and the more recently proposed allele-specific oligonucleotide-qPCR approach. ${ }^{13}$ In addition, we also investigated the presence of multilineage bone marrow involvement by the KIT D816V mutation in highly purified bone marrow $\mathrm{CD}_{3} 4^{+}$hematopoietic stem and precursor cells and maturing neutrophils using both methods. Our aim was to determine the precise frequency of peripheral blood involvement by the KIT D816V mutation, its relationship with the degree of involvement of bone marrow hematopoiesis in indolent systemic mastocytosis and the potential diagnostic implications of these findings.

\section{Materials and methods}

\section{Patients and Samples}

gDNA was obtained from bone marrow and peripheral blood samples from a total of 192 adult patients diagnosed with systemic mastocytosis $(n=190)$ and monoclonal mast cell activation syndrome $(n=2)$ that carry the KIT D816V mutation. These 192 KIT $\mathrm{D} 16 \mathrm{~V}^{+}$cases were selected from a series of 456 consecutively diagnosed systemic mastocytosis patients, of whom 417 (91\%) had the KIT D816V mutation, 9 (2\%) had other exon 17 KIT mutations, 5 (1\%) had KIT mutations in exons other than exon 17 and $25(6 \%)$ had no detectable KIT mutations. All patients were prospectively diagnosed and classified between January 2008 and December 2012 at the reference centers of the Spanish Network on Mastocytosis (REMA; Mast Cell Unit of the Hospital Virgen del Valle, Toledo, Spain, and Cytometry Service of the Cancer Research Center, Salamanca, Spain) by the WHO 2008 criteria $^{2}$ and the more recent European Competence Network on Mastocytosis ${ }^{18}$ and REMA guidelines, ${ }^{3}$ with the following distribution: indolent systemic mastocytosis with skin lesions, 135 cases; indolent systemic mastocytosis without skin lesions, 44 cases; aggressive systemic mastocytosis, 9 cases; systemic mastocytosis with an associated hematological non-mast cells lineage disease, 2 cases, and; monoclonal mast cell activation syndrome, 2 patients. Paired peripheral blood and bone marrow samples from all cases were analyzed by the peptide nucleic acid-mediated PCR method; ${ }^{17}$ all such peripheral blood samples were analyzed in parallel by the allele-specific oligonucleotide-qPCR method. Furthermore, in a subset of 161 patients (indolent systemic mastocytosis with skin lesions, 111; indolent systemic mastocytosis without skin lesions, 41; aggressive systemic mastocytosis, 6; systemic mastocytosis with an associated hematological non-mast cells lineage disease, 1; and monoclonal mast cell activation syndrome, 2 cases) in whom enough gDNA from bone marrow-purified cell populations was available, analysis on bone marrow cells was also performed in parallel by allele-specific oligonucleotide-qPCR. In addition, peripheral blood samples from a total of 35 healthy adults were analyzed in parallel by the two PCR methods. In all cases, informed consent was given by 
the donor before entering the study, according to the Declaration of Helsinki; the study was approved by the local Ethics Committees of the two centers. Overall, 64 of 192 patients included in this study have been included in previously reported series of systemic mastocytosis patients by the REMA. ${ }^{19-22}$

\section{Isolation of Bone Marrow Cell Populations}

Isolation of bone marrow mast cells, CD $34^{+}$hematopoietic stem and precursor cells, eosinophils, monocytes, maturing neutrophils and lymphocytes was performed using a four-way FACSAria III (Becton Dickinson Bioscences, San Jose, CA, USA) fluorescence-activated cell sorter (FACS) equipped with the FACSDiva software (Becton Dickinson Bioscences), as described elsewhere. ${ }^{6,8}$ The overall purity of the FACS-sorted cell populations was systematically $>96 \%$ (mean \pm 1 s.d. of $98 \pm 2 \%$ ), with $<0.01 \%$ contamination by bone marrow mast cells in all purified non-mast cell fractions.

\section{DNA Extraction and Detection of the KIT Mutation}

gDNA was extracted from the different FACSpurified bone marrow cell populations using the GenElute Mammalian Genomic DNA Miniprep (Sigma-Aldrich, St Louis, MO, USA) and the NucleoSpin Tissue XS (Macherey-Nagel, Düren, Germany), according to the manufacturer's instructions. In parallel, gDNA was also obtained from unfractionated peripheral blood samples from both systemic mastocytosis patients and healthy controls by a salting-out method (Gentra Puregene Blood kit; Qiagen, Hilden, Germany), following the standard operating procedures of the Spanish National DNA Bank Carlos III (University of Salamanca; http:// www.bancoadn.org), where gDNA from the peripheral blood samples is now publicly available.

Detection of the KIT D816V mutation was performed in gDNA from peripheral blood and highly purified bone marrow cell populations using two different methods. First, the established standard approach based on a peptide nucleic acid-mediated PCR technique (LightCycler 2.0, software version 3.5; Roche Diagnostics GmbH, Mannheim, Germany) was used, as described elsewhere. ${ }^{6,17}$ Based on the results obtained with this method on purified bone marrow cell populations, all patients were further subclassified as having either systemic mastocytosis with KIT D816V mutation restricted to bone marrow mast cells (mast cell-restricted systemic mastocytosis) or systemic mastocytosis with multilineage involvement of bone marrow hematopoiesis by the KIT mutation (multilineage systemic mastocytosis), following the previously defined criteria. ${ }^{6,10}$ In a subset of 161 patients, detection of the KIT D816V mutation was also performed in parallel in aliquots of the same samples containing the same amount of gDNA (200 ng per sample for peripheral blood leukocytes, and $50 \mathrm{ng}$ per sample for purified bone marrow neutrophils and/or CD $34^{+}$hematopoietic stem and precursor cells) using a previously described allele-specific oligonucleotide-qPCR assay; ${ }^{13}$ for this purpose, an incubation at $95^{\circ} \mathrm{C}$ for the activation of the DNA polymerase $(10 \mathrm{~min})$ followed by 45 cycles of PCR amplification (denaturation at $95^{\circ} \mathrm{C}$ for $10 \mathrm{~s}$ followed by annealing at $60^{\circ} \mathrm{C}$ for $30 \mathrm{~s}$ and elongation at $72{ }^{\circ} \mathrm{C}$ for $1 \mathrm{~s}$ ) was performed in a LightCycler 2.0 thermocycler using the LightCycler TaqMan Master kit reagents (Roche Diagnostics).

All hybridization probes required for the peptide nucleic acid-mediated PCR technique ${ }^{6}$ as well as the primers used in both techniques, were obtained from Sigma-Aldrich, whereas the allele-specific oligonucleotide-qPCR TaqMan probe $^{13}$ was purchased from Applied Biosystems (Foster City, CA, USA). As negative and positive controls, gDNA from an adult healthy donor and the KIT D816V mutation-positive HMC- $1^{560+/ 816+}$ cell line (proved to have two copies of the KIT gene, including a KIT D816V-mutated allele and a wild-type KIT allele) ${ }^{23}$ were systematically studied in parallel to both the patient and control samples. For the identification of the most discriminating cutoff to classify both negative vs positive samples, as well as cases with mast cellrestricted vs multilineage involvement of hematopoiesis by the KIT D816V mutation, receiver operating characteristic curves were applied to select for the most specific crossing threshold $\left(C_{\mathrm{t}}\right)$ value $\left(C_{\mathrm{t}}\right.$ value that provided the best sensitivity in the absence of false-positive results); $C_{\mathrm{t}}<37$ for negative vs positive samples and $C_{\mathrm{t}}<32$ for mast cell-restricted $v s$ multilineage bone marrow involvement. For those peripheral blood samples that were positive for the KIT D816V mutation, as assessed by the allelespecific oligonucleotide-qPCR method, quantification of the mutated KIT allele burden was calculated by plotting individual results against a standard curve with percentages of mutated cells ranging from 0.01 to $100 \%$, as generated through a series of dilutions of KIT D816V mutation-positive gDNA from the HMC- ${ }^{560+/ 816+}$ cell line into wild-type gDNA from a pool of healthy control samples; in turn, the allele burden for the KIT mutation in bone marrow samples was quantified by the same allelespecific oligonucleotide-qPCR assay using the 'deltadelta Ct' $\left(\Delta \Delta C_{\mathrm{t}}\right)$ method $^{24}$ to correct for the potential presence of false-negative results owing to the low amounts of gDNA obtained from bone marrowpurified cell populations. The efficiency of the $\Delta \Delta C_{\mathrm{t}}$ method was evaluated using the standard curve regression analysis (LightCycler 3.5 software) generated with gDNA from the HMC- $1^{560+/ 816+}$ cell line. Correlation of the results obtained with the two different analytical strategies used for peripheral blood vs bone marrow samples was performed in a subgroup of 90 cases, in which enough gDNA from bone marrow-purified cell populations was available, such analysis confirming that both approaches provided highly concordant results. 


\section{Statistical Methods}

For all statistical analyses, the SPSS 20.0 software (SPSS, Chicago, IL, USA) was used. The MannWhitney $U$-test and the $\chi^{2}$ tests were used to determine the statistical significance of the differences observed between groups; receiver operating characteristic curve analysis was used to identify the most discriminating cutoff for the presence of the KIT D816V mutation in peripheral blood, as assessed by the allele-specific oligonucleotide-qPCR method. Sensitivity was calculated as (true positive)/(true positive+false negative cases), specificity as (true negative)/(true negative+false positive cases) and overall accuracy as (true positive+true negative)/(true positive+false positive+true negative+false negative cases). Correlation between two variables was assessed by the Spearman's test $\left(r_{\mathrm{s}}\right)$. P-values $<0.05$ were considered to be associated with statistical significance.

\section{Results}

\section{Multilineage Involvement of Bone Marrow Cells by the} KIT D816V Mutation

All patients $(n=192)$ had the KIT D816V mutation in FACS-purified bone marrow mast cells, as assessed by the peptide nucleic acid-mediated PCR method. In a substantial fraction of cases $(67 / 192 ; 35 \%)$, the KIT mutation was also present in other bone marrow cell compartments such as the CD34+ hematopoietic stem and precursor cells, eosinophils, monocytes and maturing bone marrow neutrophils, as evaluated by the same PCR method. In addition, in 28 cases carrying multilineage KIT mutation, the KIT D816V mutation was also detected by the same technique in bone marrow $\mathrm{T}$ lymphocytes (28/192; 15\%). The relative distribution of cases carrying multilineage involvement of bone marrow hematopoiesis by the KIT mutation within the different diagnostic subtypes of systemic mastocytosis was as follows: indolent systemic mastocytosis without skin lesions, 5/44 cases (11\%); indolent systemic mastocytosis with skin lesions, 51/135 cases (37\%); aggressive systemic mastocytosis, 9/9; systemic mastocytosis with an associated hematological non-mast cell lineage disease, 2/2; and monoclonal mast cell activation syndrome, $0 / 2$.

\section{Frequency of the KIT D816V Mutation in Peripheral Blood}

Overall, none of the healthy controls analyzed (0/35) tested positive for the KIT D816V mutation in peripheral blood by any of the two PCR methods used. In contrast, the majority of mastocytosis patients were positive for this KIT mutation in peripheral blood by allele-specific oligonucleotideqPCR $(164 / 192 ; 85 \%)$ and/or by the peptide nucleic
acid-mediated-PCR technique (84/192; 44\%). Of note, all 84 cases that tested positive for the KIT D816V mutation with the peptide nucleic acidmediated PCR technique were also positive by allele-specific oligonucleotide-qPCR. These results indicate that while both methods are highly (100\%) specific, the allele-specific oligonucleotide-qPCR technique is almost two times more sensitive in peripheral blood compared with the peptide nucleic acid-mediated PCR method (85\% vs 44\%; respectively; $P<0.0001)$. Thereby, the overall accuracy of the allele-specific oligonucleotide-qPCR was clearly higher compared with that of the peptide nucleic acid-mediated PCR (88\% vs 52\%, respectively; $P<0.0001$ ) owing to its greater sensitivity (Table 1).

Despite the differences described for the two methods, their sensitivity was clearly lower among indolent systemic mastocytosis cases without skin lesions (indolent systemic mastocytosis without skin lesions; $n=44$ ) compared with those showing skin involvement (indolent systemic mastocytosis with skin lesions; $n=135): 18 \%$ vs $48 \%(P=0.0004)$ for the peptide nucleic acid-mediated-PCR and $66 \%$ vs 92\% $(P<0.0001)$ for the allele-specific oligonucleotide-qPCR, respectively. This translated into a lower overall accuracy for the detection of the KIT mutation in peripheral blood samples from indolent systemic mastocytosis without skin lesions vs indolent systemic mastocytosis with skin lesions cases for the allele-specific oligonucleotide-qPCR (81\% vs $93 \%$, respectively; $P=0.02$ ) but not for the peptide nucleic acid-mediated PCR technique (54\% vs 59\%, respectively; $P=0.60$ ) (Table 2). However, the peptide nucleic acid-mediated PCR method emerged as a more accurate approach compared with the (qualitative) allele-specific oligonucleotide-qPCR method (overall accuracy of $83 \%$ vs $48 \%$, respectively) in predicting the multilineage involvement of bone marrow hematopoiesis by the KIT mutation (Table 1), based on peripheral blood analysis when the qualitative threshold for positivity $\left(C_{\mathrm{t}}<37\right.$; corresponding to an allele burden of $0.01 \%$ ) by the allele-specific oligonucleotide-qPCR method was used. Thus, while the former method showed a lower sensitivity $(88 \%$ vs $98 \% ; P=0.02)$, it had a significantly higher specificity (80\% vs $22 \%$; $P<0.0001$ ) (Table 1). Of note, when patients $(n=161)$ were classified according to the degree of involvement of hematopoiesis by the mutation as carrying mast cell-restricted vs multilineage KIT mutation using the (qualitative) allele-specific oligonucleotide-qPCR instead of the peptide nucleic acidmediated-PCR method for the analysis of purified bone marrow maturing neutrophils, the overall accuracy of both methods was also similar (52\% vs $56 \%, P>0.05)$; despite this, the qualitative allelespecific oligonucleotide-qPCR method still showed a lower specificity compared with the peptide nucleic acid-mediated PCR method (18\% vs $63 \%$; $P<0.0001)$ in association with a significantly higher 
Table 1 Comparative analysis of the performance of the peptide nucleic acid-mediated-PCR vs the allele-specific oligonucleotide-qPCR methods $^{\mathrm{a}}$

Prediction for the KIT mutation (patients, $\mathrm{n}=192$; controls, $\mathrm{n}=35$ )

\begin{tabular}{|c|c|c|c|}
\hline & \\
\hline & $P N A$-mediated-PCR & Qualitative ASO-qPCR & $\mathrm{P}$-value \\
\hline True positive & $84 / 192(44 \%)$ & 164/192 (85\%) & \\
\hline False positive & $0 / 35(0 \%)$ & $0 / 35(0 \%)$ & \\
\hline True negative & $35 / 35(100 \%)$ & $35 / 35(100 \%)$ & \\
\hline False negative & $108 / 192(56 \%)$ & $28 / 192(15 \%)$ & \\
\hline Sensitivity & $44 \%$ & $85 \%$ & $<0.0001$ \\
\hline Specificity & $100 \%$ & $100 \%$ & NS \\
\hline Overall accuracy & $52 \%$ & $88 \%$ & $<0.0001$ \\
\hline
\end{tabular}

Prediction for multilineage KIT mutation (mast cell-restricted systemic mastocytosis, $\mathrm{n}=125$; multilineage systemic mastocytosis, $\mathrm{n}=67$ )

\begin{tabular}{|c|c|c|c|c|c|}
\hline & \multirow{2}{*}{$P N A$-mediated-PCR } & \multicolumn{4}{|c|}{$A S O-q P C R$} \\
\hline & & Qualitative $T>0.01 \%$ & $\mathrm{P}$-value & Quantitative $T>6 \%$ & $\mathrm{P}$-value \\
\hline True positive & 59/67 (88\%) & 66/67 (98\%) & & $33 / 67(49 \%)$ & \\
\hline False positive & $25 / 125(20 \%)$ & 98/125 (78\%) & & 1/125 (1\%) & \\
\hline True negative & $100 / 125(80 \%)$ & $27 / 125(22 \%)$ & & $124 / 125(99 \%)$ & \\
\hline False negative & 8/67 (12\%) & 1/67 (2\%) & & $34 / 67(51 \%)$ & \\
\hline Sensitivity & $88 \%$ & $98 \%$ & 0.02 & $49 \%$ & $<0.0001$ \\
\hline Specificity & $80 \%$ & $22 \%$ & $<0.0001$ & $99 \%$ & $<0.0001$ \\
\hline Overall accuracy & $83 \%$ & $48 \%$ & $<0.0001$ & $82 \%$ & NS \\
\hline
\end{tabular}

Abbreviations: ASO: allele-specific oligonucleotide; false negative case: case that meets the condition but tested negative; false positive case: case that does not meet the condition but tested positive; NS: not statistically significantly different; PNA: peptide nucleic acid; T: threshold.

Systemic mastocytosis patients were classified as having mast cell-restricted or multilineage KIT mutation according to the results of peptide nucleic acid-mediated PCR analysis of gDNA from purified bone marrow cell populations, as described in the Material and Methods section.

${ }^{\text {a}}$ For detection of the KIT D816V mutation in peripheral blood gDNA and prediction for the presence of the KIT D816V mutation in bone marrow mast cells and other hematopoietic cells from KIT D816V $\mathrm{V}^{+}$systemic mastocytosis $(n=190)$ and clonal mast cell activation syndrome $(n=2)$ patients.

sensitivity ( $90 \%$ vs $49 \% ; P<0.01$ ) (Table 3). Interestingly, the overall accuracy of the peptide nucleic acid-mediated PCR method evaluated in peripheral blood to predict for the presence of multilineage involvement of hematopoiesis by the KIT mutation was higher among indolent systemic mastocytosis without skin lesions (93\%) vs indolent systemic mastocytosis with skin lesions (77\%) patients $(P=0.02)$, whereas it was similar for both patient groups for the qualitative (negative vs positive peripheral blood KIT mutation) allele-specific oligonucleotide-qPCR approach $(45 \%$ vs $44 \%$, respectively) (Table 2). As expected, the peripheral blood KIT D816V-positive allele burden observed among patients with mast cell-restricted KIT mutation $(n=98)$ was significantly lower $(P<0.0001)$ compared with that of multilineage systemic mastocytosis patients $(n=66)$ with a mean percentage \pm 1 s.d. of KIT- mutated cells of $1.9 \pm 2 \%$ vs $15 \pm 20 \%$, respectively (Figure 1a). Thereby, when the most specific cutoff (corresponding to an allele burden of $6 \%$ by the allele-specific oligonucleotide-qPCR method) was selected, to increase the specificity for the discrimination between mast cell-restricted vs multilineage systemic mastocytosis cases through analysis of peripheral blood samples by the allelespecific oligonucleotide-qPCR method, a similar overall accuracy was obtained for the quantitative allele-specific oligonucleotide-qPCR vs the peptide nucleic acid-mediated PCR method (82\% vs $83 \%$; $P>0.05)$. Nevertheless, it should be noted that based on the KIT D816V allele burden of $6 \%$ by quantitative allele-specific oligonucleotide-qPCR virtually all mast cell-restricted systemic mastocytosis patients were correctly classified, whereas only half (33/67) of all multilineage systemic mastocytosis patients were identified as such (49\% sensitivity; 99\% specificity) (Figure 1a). Similar results were observed once indolent systemic mastocytosis cases were subdivided into indolent systemic mastocytosis without skin lesions and indolent systemic mastocytosis with skin lesions patients: $60 \%$ and $44 \%$ sensitivity and $99 \%$ and $98 \%$ specificity, and a $93 \%$ and $76 \%$ overall accuracy, respectively (Figure $1 \mathrm{~b}$ ).

\section{Degree of Involvement of Distinct Bone Marrow Cell Compartments from Systemic Mastocytosis Patients by the KIT D816V Mutation as Defined by the Allele-Specific Oligonucleotide-qPCR vs the Peptide Nucleic Acid-Mediated-PCR Techniques}

In a subset of 152/179 indolent systemic mastocytosis patients, in whom enough gDNA from purified 
Table 2 Detection of the KIT mutation in peripheral blood gDNA from indolent systemic mastocytosis patients $(n=179)$ grouped according to the presence vs absence of skin lesions by the peptide nucleic acid-mediated-PCR vs the allele-specific oligonucleotide-qPCR assays $^{\mathrm{a}}$

Prediction for the KIT mutation

\begin{tabular}{|c|c|c|c|c|c|c|}
\hline & \multicolumn{3}{|c|}{$\begin{array}{l}\text { Indolent systemic mastocytosis } \\
\text { without skin lesions }{ }^{\mathrm{b}}(\mathrm{n}=44)\end{array}$} & \multicolumn{3}{|c|}{$\begin{array}{l}\text { Indolent systemic mastocytosis } \\
\text { with skin lesions }(\mathrm{n}=135)\end{array}$} \\
\hline & $P N A-P C R$ & $\begin{array}{l}\text { Qualitative } \\
\text { ASO-qPCR }\end{array}$ & $\mathrm{P}$-value & $P N A-P C R$ & $\begin{array}{l}\text { Qualitative } \\
\text { ASO-qPCR }\end{array}$ & $\mathrm{P}$-value \\
\hline Sensitivity & $18 \%$ & $66 \%$ & $<0.0001$ & $48 \%$ & $92 \%$ & $<0.0001$ \\
\hline Specificity & $100 \%$ & $100 \%$ & NS & $100 \%$ & $100 \%$ & NS \\
\hline Overall accuracy & $54 \%$ & $81 \%$ & 0.007 & $59 \%$ & $93 \%$ & $<0.0001$ \\
\hline
\end{tabular}

Prediction for multilineage KIT mutation

\begin{tabular}{|c|c|c|c|c|c|c|c|c|c|c|}
\hline & \multicolumn{5}{|c|}{$\begin{array}{l}\text { Indolent systemic mastocytosis } \\
\text { without skin lesions }{ }^{b}(\mathrm{n}=44)\end{array}$} & \multicolumn{5}{|c|}{$\begin{array}{l}\text { Indolent systemic mastocytosis } \\
\text { with skin lesions }{ }^{c}(\mathrm{n}=135)\end{array}$} \\
\hline & \multirow{2}{*}{$P N A-P C R$} & \multicolumn{4}{|c|}{ ASO-qPCR } & \multirow{2}{*}{$P N A-P C R$} & \multicolumn{4}{|c|}{ ASO-qPCR } \\
\hline & & $\begin{array}{c}\text { Qualitative } \\
T>0.01 \%\end{array}$ & $\mathrm{P}$-value & $\begin{array}{c}\text { Quantitative } \\
T>6 \%\end{array}$ & $\mathrm{P}$-value & & $\begin{array}{c}\text { Qualitative } \\
T>0.01 \%\end{array}$ & P-value & $\begin{array}{c}\text { Quantitative } \\
T>6 \%\end{array}$ & P-value \\
\hline Sensitivity & $100 \%$ & $100 \%$ & NS & $60 \%$ & NS & $84 \%$ & $98 \%$ & 0.01 & $44 \%$ & $<0.0001$ \\
\hline Specificity & $92 \%$ & $38 \%$ & $<0.0001$ & $100 \%$ & NS & $74 \%$ & $12 \%$ & $<0.0001$ & $98 \%$ & $<0.0001$ \\
\hline Overall accuracy & $93 \%$ & $45 \%$ & $<0.0001$ & $93 \%$ & NS & $77 \%$ & $44 \%$ & $<0.0001$ & $76 \%$ & NS \\
\hline
\end{tabular}

Abbreviations: ASO: allele-specific oligonucleotide; NS: not statistically significantly different; PNA-PCR: peptide nucleic acid-mediated PCR; T: threshold.

Systemic mastocytosis patients were classified as having mast cell-restricted or multilineage KIT mutation according to the results of peptide nucleic acid-mediated PCR analysis of gDNA from purified bone marrow cell populations, as described in the Material and Methods section. All aggressive systemic mastocytosis cases $(n=9)$ and systemic mastocytosis with an associated hematological non-mast cell lineage disease $(n=2)$ had multilineage KIT mutation and they were all positive in peripheral blood gDNA by the two methods. Conversely, the two monoclonal mast cell activation syndrome patients showed mast cell-restricted KIT mutation in the bone marrow and they were both negative in peripheral blood by the two methods evaluated.

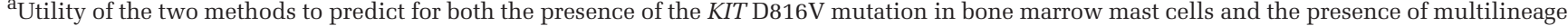
bone marrow involvement by the KIT mutation.

b Thirty-nine indolent systemic mastocytosis without skin lesions cases had mast cell-restricted KIT mutation and five showed multilineage involvement of hematopoiesis.

${ }^{\mathrm{c}}$ Eighty-four indolent systemic mastocytosis with skin lesion cases had mast cell-restricted KIT mutation and 51 multilineage KIT mutation.

Table 3 Performance of the peptide nucleic acid-mediated-PCR vs the allele-specific oligonucleotide-qPCR methods in detecting the KIT mutation in peripheral blood gDNA and predicting for the presence of multilineage KIT mutation by allele-specific oligonucleotide-qPCR analysis of purified bone marrow maturing neutrophils

Prediction for multilineage KIT mutation(mast cell-restricted systemic mastocytosis, $\mathrm{n}=84$; multilineage systemic mastocytosis, $\mathrm{n}=77$ )

\begin{tabular}{|c|c|c|c|c|c|}
\hline & \multirow{2}{*}{ PNA-mediated-PCR } & \multicolumn{4}{|c|}{$A S O-q P C R$} \\
\hline & & Qualitative $T>0.01 \%$ & P-value & Quantitative $T>6 \%$ & P-value \\
\hline True positive & $38 / 77(49 \%)$ & $69 / 77(90 \%)$ & & $19 / 77(25 \%)$ & \\
\hline False positive & $31 / 84(37 \%)$ & $69 / 84(82 \%)$ & & 7/84 (8\%) & \\
\hline True negative & $53 / 84(63 \%)$ & $15 / 84(18 \%)$ & & $77 / 84(92 \%)$ & \\
\hline False negative & $39 / 77(51 \%)$ & $8 / 77(10 \%)$ & & $58 / 77(75 \%)$ & \\
\hline Sensitivity & $49 \%$ & $90 \%$ & 0.01 & $25 \%$ & 0.002 \\
\hline Specificity & $63 \%$ & $18 \%$ & $<0.0001$ & $92 \%$ & $<0.0001$ \\
\hline Overall accuracy & $56 \%$ & $52 \%$ & NS & $60 \%$ & NS \\
\hline
\end{tabular}

Abbreviations: ASO: allele-specific oligonucleotide; false negative case: case that meets the condition but tested negative; false positive case: case that does not meet the condition but tested positive; NS: not statistically significantly different; PNA: peptide nucleic acid; T: threshold. 

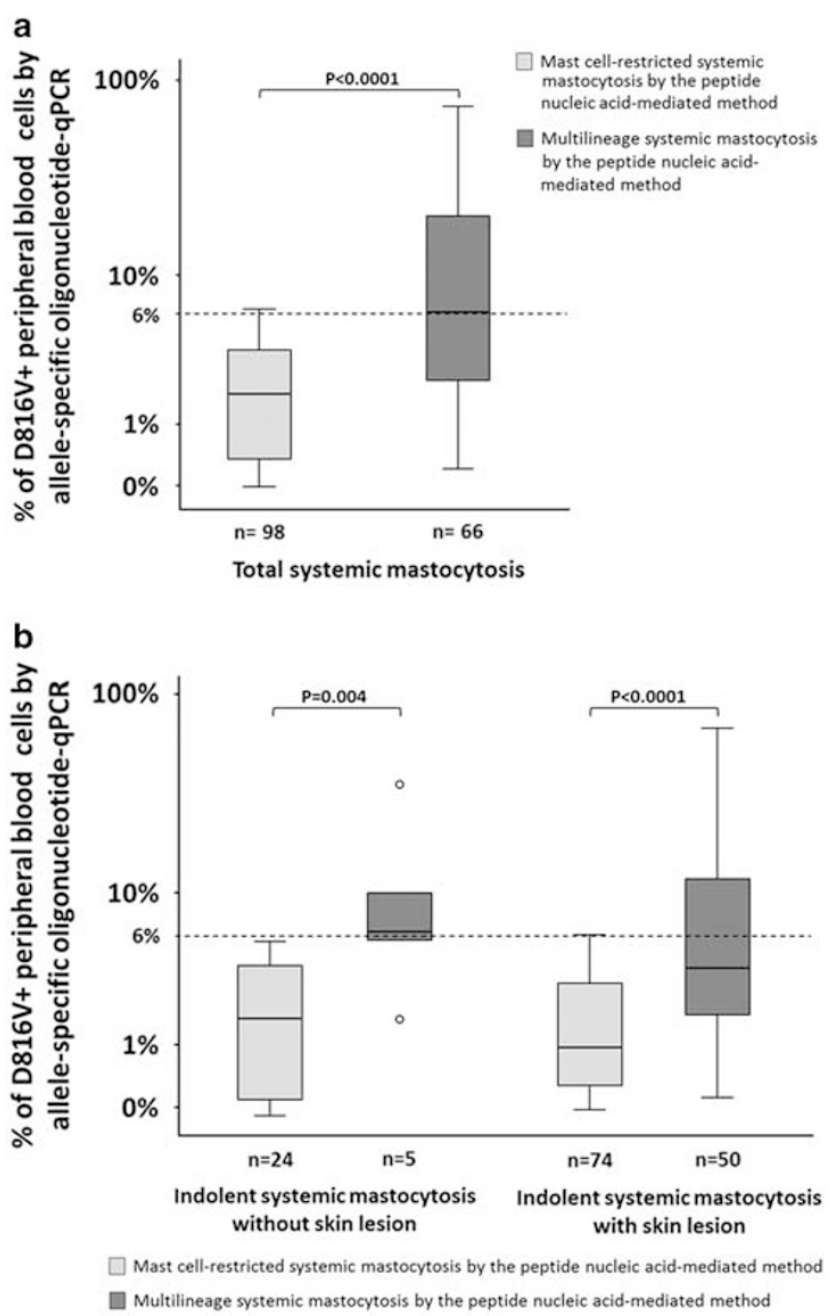

Figure 1 KIT D816V-positive allele burden in peripheral blood leukocytes from systemic mastocytosis patients, as assessed by allele-specific oligonucleotide-qPCR. The percentage of KIT D816 $\mathrm{V}^{+}$peripheral blood leukocytes grouped according to the degree of bone marrow involvement by the KIT mutation as defined by the conventional peptide nucleic acid-mediated-PCR technique is displayed. In (a), the percentage of KIT-positive peripheral blood leukocytes in the overall group of systemic mastocytosis patients is shown, whereas in (b), the percentage of KIT-positive peripheral blood leukocytes among indolent systemic mastocytosis without skin lesions and indolent systemic mastocytosis with skin lesions patients is displayed. Notched boxes extend from the 25th to 75th percentile values; the lines in the middle and vertical lines correspond to median values and the 10th and 90th percentiles, respectively, whereas the horizontal dotted line corresponds to the $6 \%$ cutoff allele burden by allelespecific oligonucleotide-qPCR.

bone marrow $\mathrm{CD}_{34}{ }^{+}$hematopoietic stem and precursor cells and/or maturing neutrophils was available, we investigated the frequency of cases showing the KIT D816 $\mathrm{V}^{+}$mutation in both purified bone marrow cell populations also using the allelespecific oligonucleotide-qPCR method (all these cases had already been tested and classified as having mast cell-restricted or multilineage involvement of bone marrow hematopoiesis by the KIT mutation by the peptide nucleic acid-mediated PCR method). Overall, allele-specific oligonucleotideqPCR showed the presence of the KIT mutation in purified $\mathrm{CD} 4^{+}$hematopoietic stem and precursor cells and maturing neutrophils from 76/105 (72\%) and $71 / 152(46 \%)$ indolent systemic mastocytosis cases analyzed, respectively (Figure 2a). In detail, virtually all cases $(39 / 43 ; 93 \%)$ who were classified by the peptide nucleic acid-mediated PCR assay as having multilineage involvement of hematopoiesis by the KIT mutation showed involvement of CD34 ${ }^{+}$ hematopoietic stem and precursor cells (28/30, 93\%) and/or maturing neutrophils $(25 / 43,58 \%)$ also by the allele-specific oligonucleotide-qPCR technique. Additionally, a great proportion of cases (73/109; $67 \%$ ) with mast cell-restricted bone marrow involvement by the KIT mutation, as assessed by the peptide nucleic acid-mediated PCR method, also showed involvement of $\mathrm{CD}^{+} 4^{+}$hematopoietic stem and precursor cells $(48 / 75 ; 64 \%)$ and/or maturing neutrophils $(46 / 109 ; 42 \%)$ by the KIT mutation by the allele-specific oligonucleotide-qPCR assay. As expected, lower frequencies of $K I T \mathrm{D}_{16} 16 \mathrm{~V}^{+} \mathrm{CD} 34^{+}$ hematopoietic stem and precursor cells (mean \pm 1 s.d. of $6.6 \%$ vs $21 \pm 26 \%$; $P=0.001$ ) and maturing neutrophils (mean \pm 1 s.d. of $17 \pm 32 \%$ vs $31 \% \pm 35 \%$; $P=0.0003$ ) were observed with the allele-specific oligonucleotide-qPCR technique among those positive cases that showed mast cell-restricted vs multilineage involvement of hematopoiesis by the peptide nucleic acid-mediated-PCR method (Figure 2b).

\section{Correlation Between the Percentage of KIT D816V- Mutated Bone Marrow CD34 ${ }^{+}$Hematopoietic Stem and Precursor Cells and Maturing Neutrophils and that of Peripheral Blood Leukocytes as Detected by Allele- Specific Oligonucleotide-qPCR}

Overall, no significant correlation $(P>0.05)$ was observed between the percentage of bone marrow CD $34^{+}$hematopoietic stem and precursor cells and maturing neutrophils carrying the KIT D816V mutation by allele-specific oligonucleotide-qPCR, when all cases were analyzed together (Figure 3a); however, this correlation increased towards statistical significance $\left(r_{\mathrm{s}}=0.61 ; P=0.007\right)$ when the analysis was restricted to those cases that simultaneously showed the mutation in the above two cell populations (Figure 3b). Most interestingly, only a marginally statistically significant correlation was found between the percentage of bone marrow maturing neutrophils and the percentage of peripheral blood leukocytes carrying the KIT mutation, when all cases were analyzed together $\left(r_{\mathrm{s}}=0.33 ; P=0.02\right)$ (Figure 3c); once again, when we restricted the analysis to those cases that showed the KIT mutation in both cell populations, the degree of correlation increased $\left(r_{\mathrm{s}}=0.60 ; P=0.002\right)$ (Figure $\left.3 \mathrm{~d}\right)$. In turn, a marginal degree of correlation was observed between the level of involvement of bone marrow CD $34^{+}$ hematopoietic stem and precursor cells and that of 

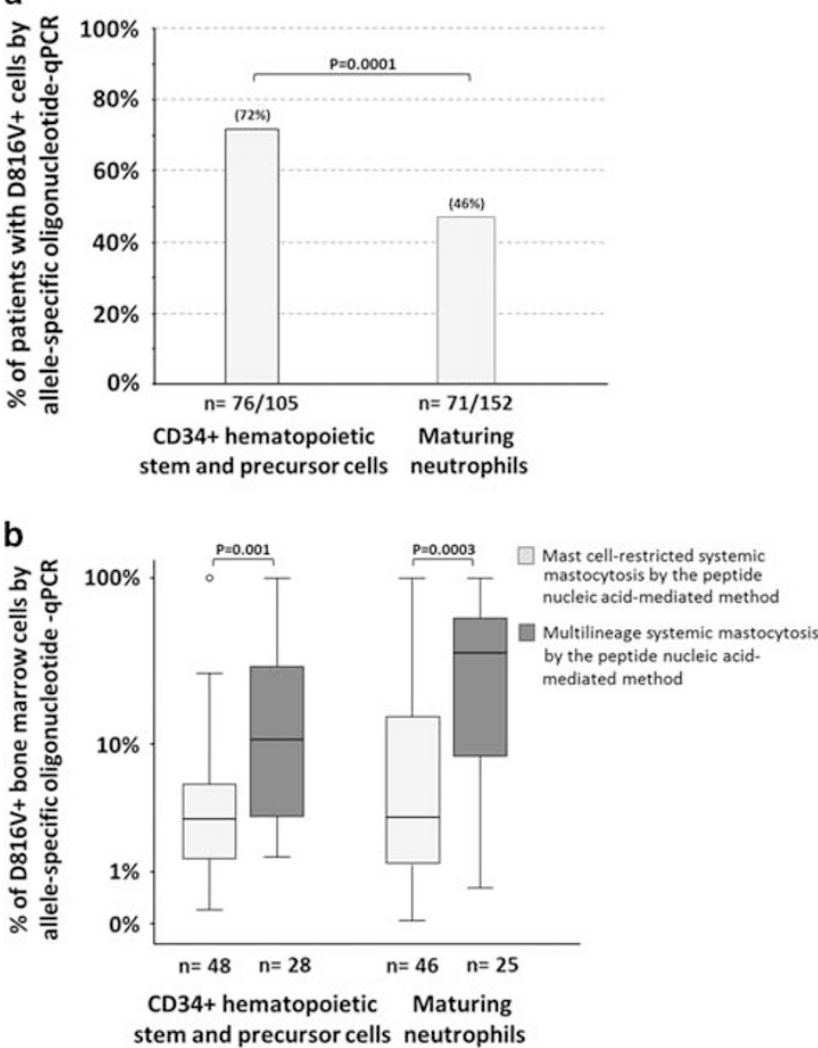

Figure 2 Frequency and degree of involvement of bone marrow isolated cells from indolent systemic mastocytosis patients grouped according to the presence of mast cell-restricted vs multilineage involvement of hematopoiesis by the KIT $\mathrm{D} 816 \mathrm{~V}^{+}$as assessed by the allele-specific oligonucleotide-qPCR method. Frequency and degree of involvement by the KIT D $816 \mathrm{~V}$ mutation of FACS-purified bone marrow CD $34^{+}$hematopoietic stem and precursor cells and maturing neutrophils from indolent systemic mastocytosis patients as assessed by the allele-specific oligonucleotide-qPCR technique. In (a), the overall frequency of indolent systemic mastocytosis cases carrying the KIT D816V mutation in bone marrow $\mathrm{CD} 34^{+}$hematopoietic stem and precursor cells and maturing neutrophils by the allele-specific oligonucleotide-qPCR method is shown, whereas in (b), the percentage of bone marrow $\mathrm{CD}^{+} 4^{+}$hematopoietic stem and precursor cells and maturing neutrophils carrying the KIT D816V mutation by allele-specific oligonucleotide-qPCR among indolent systemic mastocytosis cases who tested positive for the mutation by this technique, grouped according to the degree of bone marrow involvement by the KIT mutation as defined by the conventional peptide nucleic acid-mediated-PCR technique is displayed. Notched boxes extend from the 25th to 75th percentile values; the lines in the middle and vertical lines correspond to median values and the 10th and 90th percentiles, respectively.

peripheral blood leukocytes, both when we analyzed all cases together $\left(r_{\mathrm{s}}=0.34 ; P=0.02\right)$ (Figure 3e) and only those cases where the two cell populations were simultaneously positive for the KIT D816V mutation $\left(r_{\mathrm{s}}=0.25 ; P=0.16\right)$ (Figure $\left.3 f\right)$.

\section{Discussion}

Currently, it is well established that $\sim 90 \%$ of adult systemic mastocytosis patients carry the KIT D816V mutation in bone marrow mast cells, ${ }^{6,10}$ as also found in our series of 456 cases from which the 192 KIT D816V patients here reported were selected. Moreover, virtually all aggressive systemic mastocytosis cases, as well as around one-fourth of indolent systemic mastocytosis patients, ${ }^{6}$ present multilineage involvement of bone marrow cell compartments other than mast cells by the KIT mutation,11,12,25,26 this being considered as the most powerful prognostic factor for disease progression among adult indolent systemic mastocytosis patients. ${ }^{7,10,27}$

Recently, several groups ${ }^{9,13,14}$ have confirmed the feasibility of detecting the KIT mutation in peripheral blood leukocytes; consequently, sensitive allele-specific oligonucleotide-qPCR approaches for peripheral blood detection of the KIT D816V mutation ${ }^{13,28}$ have been included in recent consensus diagnostic algorithms ${ }^{18}$ owing to the less invasive diagnostic approach in peripheral blood vs bone marrow. However, careful analysis of the literature shows discrepant results regarding both the frequency of systemic mastocytosis cases who are KIT $\mathrm{D} 16 \mathrm{~V}^{+}$in peripheral blood and the prognostic impact of the KIT D816V allele burden. Thus, while Kristensen et al. ${ }^{9,29}$ reported positive rates in peripheral blood samples of indolent systemic mastocytosis cases between 96\% and 100\%, Erben et $a .^{30}$ only found $46 \%$ of indolent systemic mastocytosis patients to be positive in peripheral blood using a different allele-specific quantitative real-time PCR method. In turn, whereas Erben et al. ${ }^{30}$ suggested that a greater amount of KIT D816 $\mathrm{V}^{+}$cells in peripheral blood is associated with a poorer outcome, the Danish group ${ }^{15}$ did not find any correlation between the allele burden for the KIT D816V mutation and the clinical manifestations of indolent systemic mastocytosis. Overall, such apparently discrepant results could be explained by the fact that different approaches were used to assess peripheral blood involvement by the KIT mutation (analysis on gDNA $^{9,13}$ vs $\mathrm{CDNA}^{30}$ ), which therefore might have different sensitivities. Of note, no study has been reported so far in which paired peripheral blood and bone marrow samples had been analyzed in large series of systemic mastocytosis cases by two or more (highly sensitive) PCR methods, ${ }^{28}$ to clarify such discrepancies. Furthermore, the precise relationship between the presence of KIT $\mathrm{D} 816 \mathrm{~V}^{+}$cells in peripheral blood and multilineage involvement of bone marrow hematopoiesis by the KIT mutation also remains unknown.

In this study, we compared the performance of the two most widely accepted methods ${ }^{28}$ (peptide nucleic acid-mediated PCR-clamping technique ${ }^{17}$ vs allele-specific oligonucleotide-qPCR ${ }^{13}$ ), which are currently used to detect the KIT D816V mutation in clinical diagnostic laboratories, on the same gDNA samples obtained from paired peripheral blood leukocytes and highly purified bone marrow CD $34^{+}$ hematopoietic stem and precursor cells and maturing neutrophils, from a large cohort of systemic 
a

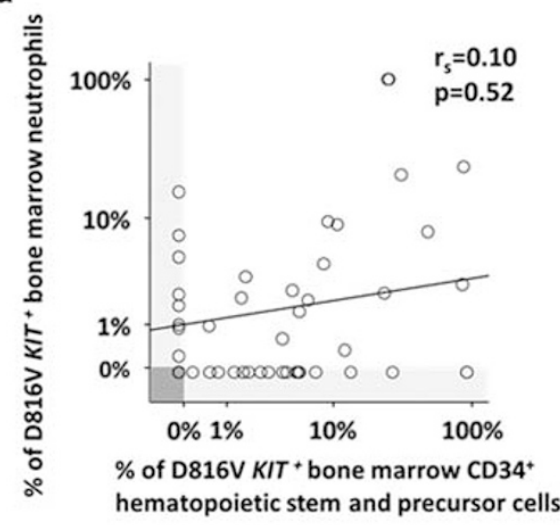

C

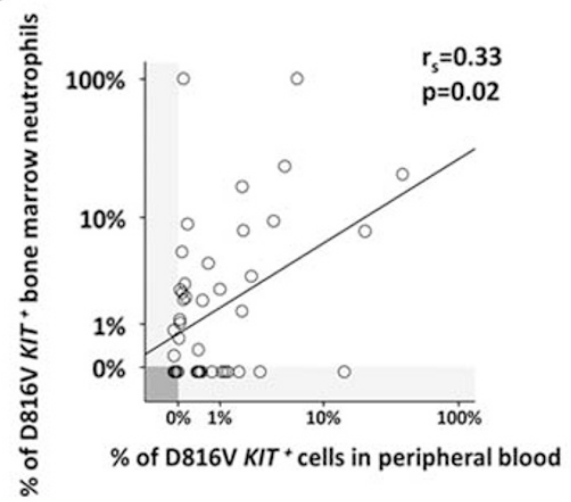

e

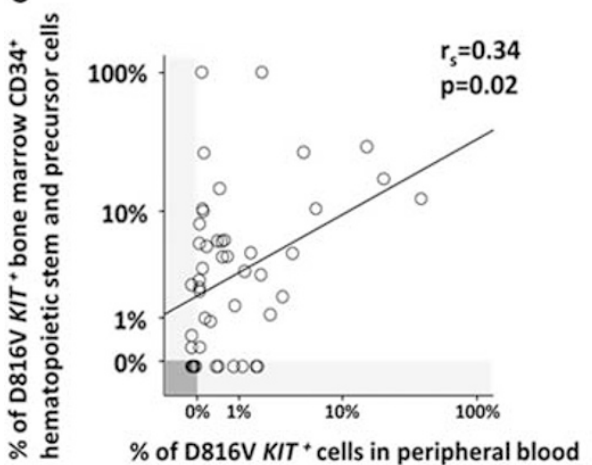

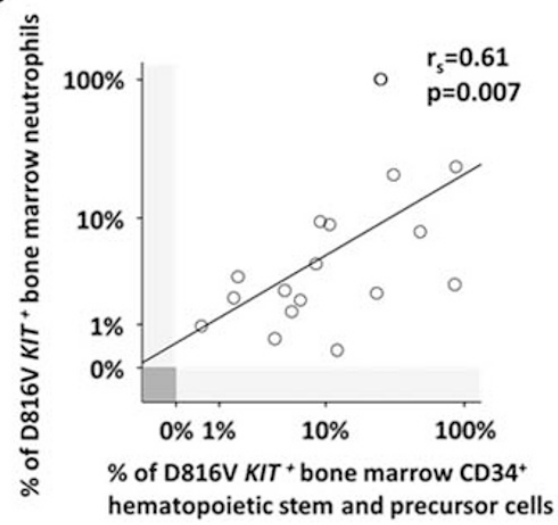

d

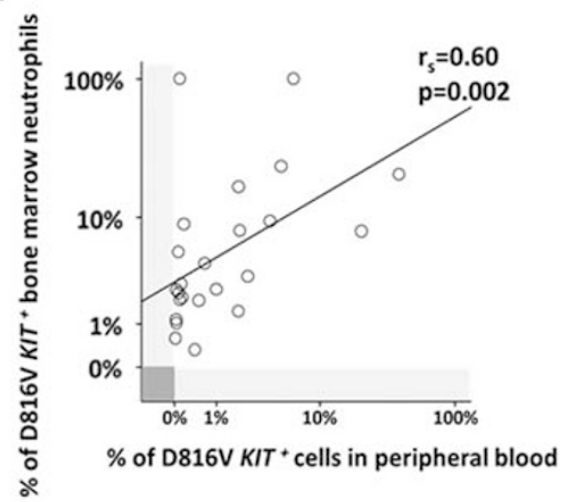

f

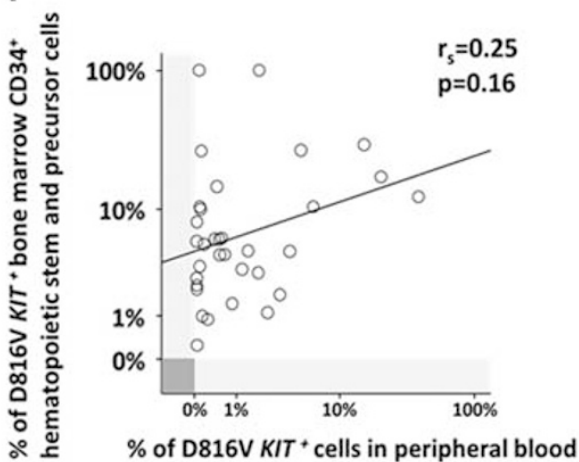

Figure 3 Correlation between the percentage of KIT D816V mutated bone marrow CD34 ${ }^{+}$hematopoietic stem and precursor cells and maturing neutrophils and peripheral blood leukocytes in systemic mastocytosis patients. Analysis of the KIT D816V mutation is shown for all indolent systemic mastocytosis patients for whom paired bone marrow CD34 ${ }^{+}$hematopoietic stem and precursor cells plus maturing neutrophils and peripheral blood leukocytes were studied $(n=49)$. In $(\mathbf{a})$ and $(\mathbf{b})$, the correlation between the percentage of KIT D816V bone marrow CD34 ${ }^{+}$hematopoietic stem and precursor cells (Y axis) and maturing neutrophils (X axis) is shown for all cases evaluated (a) and only for those patients who had the D816V mutation in both bone marrow cell subsets $(n=18)$, respectively (b). (c) and (d) The correlation between the percentage of KIT-mutated peripheral blood nucleated cells (X axis) and bone marrow-purified CD34 ${ }^{+}$ hematopoietic stem and precursor cells (Y axis) for all cases tested $(n=49)$ and for those cases who were D816V positive in both cell populations $(n=24)$, respectively. (e) and (f) The correlation between the percentage of KIT-mutated peripheral blood nucleated cells (X axis) and maturing neutrophils (Y axis) for all cases evaluated $(n=49)$ and for those cases who were D816V-positive in both cell populations $(n=33)$, respectively.

mastocytosis patients. To the best of our knowledge, this is the only series of systemic mastocytosis patients in which both methods have been performed in parallel to evaluate their accuracy in both detecting the KIT mutation in peripheral blood and determining its relationship with multilineage involvement of bone marrow hematopoiesis.

Overall, our results confirmed that allele-specific oligonucleotide-qPCR is significantly more sensitive than peptide nucleic acid-mediated PCR to identify 
the KIT D816V mutation in both peripheral blood leukocytes and purified bone marrow cell populations, in line with the higher frequencies of KIT mutated cases reported in the literature with the former technique.9,29 In contrast, both methods showed an overall similar accuracy to predict for mast cell-restricted vs multilineage bone marrow involvement by the KIT mutation based on peripheral blood screening, whenever quantitative (the KIT allele burden) instead of qualitative (negative vs positive peripheral blood for the KIT mutation) allele-specific oligonucleotide-qPCR results were used. Of note, similar results were observed when we restricted the analysis to indolent systemic mastocytosis patients grouped according to the presence vs absence of skin lesions, although both a lower sensitivity and overall accuracy for the detection of the KIT D816V mutation were observed among indolent systemic mastocytosis without skin lesions vs indolent systemic mastocytosis with skin lesions cases for the qualitative allele-specific oligonucleotide-qPCR vs both the allele-specific oligonucleotide-qPCR allele burden and the peptide nucleic acid-mediated PCR method. Thus, while the quantitative allele-specific oligonucleotide-qPCR and the peptide nucleic acid-mediated-PCR methods showed a great efficiency in classifying indolent systemic mastocytosis with skin lesions and indolent systemic mastocytosis without skin lesions patients as having mast cell-restricted vs multilineage bone marrow disease, the qualitative allele-specific oligonucleotide-qPCR approach showed a similarly poorer performance in the two subgroups of indolent systemic mastocytosis patients owing to its lower specificity. Noteworthy, similar results were also obtained when multilineage involvement of bone marrow hematopoiesis was defined by the presence of KIT-mutated bone marrow maturing neutrophils by allele-specific oligonucleotide-qPCR. Altogether, these results support the recent consensus proposal, to introduce the use of allele-specific oligonucleotide-qPCR analysis of peripheral blood leukocytes in the diagnostic screening of systemic mastocytosis. ${ }^{9,18}$ However, caution should be taken in systemic mastocytosis cases presenting with mast cell activation-associated symptoms (e.g., anaphylaxis) in the absence of skin lesions if the mutation is not detected, because around one-third of indolent systemic mastocytosis without skin lesions cases carrying the KIT D816V mutation in bone marrow cells this mutation could not be detected in peripheral blood by either of the methods. More importantly, no clear association was found between the qualitative allele-specific oligonucleotide-qPCR method and multilineage involvement of bone marrow hematopoiesis using the presence of the mutation in peripheral blood; in contrast, the KIT allele burden by (quantitative) allele-specific oligonucleotide-qPCR and the detection of the KIT D816V $\mathrm{V}^{+}$mutation in peripheral blood cells by the peptide nucleic acid-mediated PCR method were both highly predictive for multilineage involvement of bone marrow hematopoiesis.

To clarify the lack of correlation between the qualitative allele-specific oligonucleotide-qPCR and peptide nucleic acid-mediated PCR methods, we investigated the presence of the KIT mutation in highly purified compartments of bone marrow CD $34^{+}$hematopoietic stem and precursor cells and maturing neutrophils with both techniques, and compared them with the peripheral blood results. As expected, the allele-specific oligonucleotideqPCR method detected involvement of CD $34^{+}$hematopoietic stem and precursor cells and/or maturing neutrophils in a higher proportion of cases compared with the peptide nucleic acid-mediated PCR method, but with a lower median allele burden in the negative vs positive cases for the latter approach. Altogether, these results support the established notion that systemic mastocytosis is a stem cell disease, ${ }^{6,12,31}$ a notion that is further demonstrated here by the high rate of involvement of CD $34^{+}$ hematopoietic stem and precursor cells and other non-mast cells lineages by the KIT D816V mutation. Nonetheless, no significant correlation was found between the percentage of bone marrow CD $34^{+}$ hematopoietic stem and precursor cells, as well as maturing neutrophils carrying the KIT D816V mutation, with a significant proportion of cases showing the mutation in $\mathrm{CD}_{4} 4^{+}$hematopoietic stem and precursor cells but not in maturing neutrophils. This suggests that the presence of the KIT mutation in a small percentage of $\mathrm{CD} 34^{+}$hematopoietic stem and precursor cells could most likely be associated with maturation towards cell lineages other than the neutrophil lineage, for example, mast cells and potentially also other minor myeloid lineages such as the eosinophil and basophil lineages. ${ }^{12,32}$ In line with this hypothesis, although a significant correlation was found between the percentage of bone marrow maturing neutrophils (and CD34+ hematopoietic stem and precursor cells) and the proportion of peripheral blood leukocytes carrying the KIT mutation, such correlation was only marginal. These results further suggest that cell compartments other than the neutrophils and CD34 ${ }^{+}$hematopoietic stem and precursor cells also contribute to the peripheral blood allele burden. Alternatively, the lack of association between the presence of the KIT mutation in bone marrow CD34+ hematopoietic stem and precursor cells and maturing neutrophils or peripheral blood leukocytes could be due to contamination of bone marrow $\mathrm{CD}^{+} 4^{+}$hematopoietic stem and precursor cells samples by low numbers of bone marrow mast cells carrying the KIT mutation, which seems unlikely since this was systematically ruled out for all sorted cell populations.

Altogether, these results might also contribute to explain the different degree of association observed between each of the two (peptide nucleic acidmediated PCR and qualitative allele-specific oligonucleotide-qPCR) methods and multilineage bone 
marrow involvement by the KIT mutation. Thus, the KIT-mutated cell fractions detected by the allelespecific oligonucleotide-qPCR method could consist of minor circulating peripheral blood cells such as $\mathrm{CD}^{+} 4^{+}$hematopoietic stem and precursor cells, circulating mast cells progenitors and/or basophils, which were not systematically investigated to define multilineage bone marrow involvement by the peptide nucleic acid-mediated PCR method. At the same time, our results suggest that a minimum number of mutated cells circulating in peripheral blood may be required to identify bone marrow multilineage involvement in peripheral blood, as suggested by the better results found once the qualitative results of the allele-specific oligonucleotide-qPCR technique were replaced by a quantitative (allele burden) threshold. In line with this, Hoermann et al. ${ }^{14}$ recently showed that systemic mastocytosis with a higher bone marrow allele burden $(>2 \%)$ have a greater probability of showing disease progression. However, it should be noted that, despite the higher allele burden for the KIT mutation observed in our series in the peripheral blood of cases showing multilineage vs mast cellrestricted KIT mutation, still half of systemic mastocytosis patients who showed multilineage bone marrow involvement had KIT allele burden values in peripheral blood below the most specific cutoff identified ( $6 \%$ KIT-mutated peripheral blood cells). These results did not allow us to validate the peripheral blood allele burden $(6 \%)$ to stratify systemic mastocytosis patients according to their probability of showing multilineage bone marrow involvement by the KIT mutation. Therefore, further studies in large series of indolent systemic mastocytosis patients with a long follow-up are required to establish the prognostic value of the peripheral blood allele burden.

In summary, our results confirm the presence of the KIT mutation in peripheral blood of most systemic mastocytosis patients by the allele-specific oligonucleotide-qPCR method, particularly among indolent systemic mastocytosis with skin lesions and aggressive systemic mastocytosis cases, and also in the majority of indolent systemic mastocytosis without skin lesions patients. In addition, we also show that the allele burden of KIT-mutated peripheral blood cells by this method or the established peptide nucleic acid-mediated PCR approach can accurately predict for the presence of multilineage involvement of bone marrow hematopoiesis by the KIT mutation and may therefore be used as a surrogate marker for multilineage disease.

\section{Acknowledgments}

This work was supported in part by grants from the Fondo de Investigaciones Sanitarias (FIS; grant number PI11/02399, FEDER) and Red Temática de Investigación Cooperativa en Cancer (RTICC; grant number RD12/0036/0048, FEDER) of the Instituto de Salud Carlos III (Ministry of Economy and Competitivity, Madrid, Spain), from Fundacion Ramon Areces (Madrid, Spain; grant number CIVP16A1806) and from Ayudas a Proyectos de Investigación en Salud de la Fundación Mutua Madrileña 2014 and Asociación Española de Enfermos de Mastocitosis (AEDM 2014). The National DNA Bank is supported by grants from the Instituto de Salud Carlos III of the Ministerio de Economia y Competitividad of Spain (grand numbers PT13/0001/0037 and PT13/0010/ 0067, FEDER). AM was supported by RTICC.

\section{Disclosure/conflict of interest}

The authors declare no conflict of interest.

\section{References}

1 Valent P, Horny HP, Escribano L et al. Diagnostic criteria and classification of mastocytosis: a consensus proposal. Leuk Res 2001;25:603-625.

2 Horny HP, Metcalfe DD, Bennet JM et al. Mastocytosis. In: Swerdlow SHCE, Harris NL, Jaffe ES, Pileri SA, Stein H, Thiele J, Vardiman JW (eds). WHO Classification of Tumours of Haematopoietic and Lymphoid Tissues. IARC Press: Lyon, France, 2008, pp 54-63.

3 Sanchez-Munoz L, Alvarez-Twose I, Garcia-Montero $\mathrm{AC}$ et al. Evaluation of the WHO criteria for the classification of patients with mastocytosis. Mod Pathol 2011;24:1157-1168.

4 Alvarez-Twose I, Gonzalez de Olano D, SanchezMunoz L et al. Clinical, biological, and molecular characteristics of clonal mast cell disorders presenting with systemic mast cell activation symptoms. J Allergy Clin Immunol 2010;125:1269-1278.

5 Alvarez-Twose I, Gonzalez-de-Olano D, SanchezMunoz L et al. Validation of the REMA score for predicting mast cell clonality and systemic mastocytosis in patients with systemic mast cell activation symptoms. Int Arch Allergy Immunol 2012;157: 275-280.

6 Garcia-Montero AC, Jara-Acevedo M, Teodosio C et al. KIT mutation in mast cells and other bone marrow hematopoietic cell lineages in systemic mast cell disorders: a prospective study of the Spanish Network on Mastocytosis (REMA) in a series of 113 patients. Blood 2006;108:2366-2372.

7 Teodosio C, Garcia-Montero AC, Jara-Acevedo M et al. Mast cells from different molecular and prognostic subtypes of systemic mastocytosis display distinct immunophenotypes. J Allergy Clin Immunol 2010;125: 719-726.

8 Escribano L, Garcia Montero AC, Nunez R et al. Flow cytometric analysis of normal and neoplastic mast cells: role in diagnosis and follow-up of mast cell disease. Immunol Allergy Clin N Am 2006;26: 535-547.

9 Kristensen T, Vestergaard H, Bindslev-Jensen C et al. Sensitive KIT D816V mutation analysis of blood as a diagnostic test in mastocytosis. Am J Hematol 2014;89: 493-498. 
10 Escribano L, Alvarez-Twose I, Sanchez-Munoz L et al. Prognosis in adult indolent systemic mastocytosis: a long-term study of the Spanish Network on Mastocytosis in a series of 145 patients. J Allergy Clin Immunol 2009;124:514-521.

11 Akin C, Kirshenbaum AS, Semere T et al. Analysis of the surface expression of c-kit and occurrence of the c-kit Asp816Val activating mutation in T cells, B cells, and myelomonocytic cells in patients with mastocytosis. Exp Hematol 2000;28:140-147.

12 Yavuz AS, Lipsky PE, Yavuz S et al. Evidence for the involvement of a hematopoietic progenitor cell in systemic mastocytosis from single-cell analysis of mutations in the c-kit gene. Blood 2002;100:661-665.

13 Kristensen T, Vestergaard H, Moller MB. Improved detection of the KIT D $816 \mathrm{~V}$ mutation in patients with systemic mastocytosis using a quantitative and highly sensitive real-time qPCR assay. J Mol Diagn 2011;13: 180-188.

14 Hoermann G, Gleixner KV, Dinu GE et al. The KIT D816V allele burden predicts survival in patients with mastocytosis and correlates with the WHO type of the disease. Allergy 2014;69:810-813.

15 Broesby-Olsen S, Kristensen T, Vestergaard $\mathrm{H}$ et al. KIT D816V mutation burden does not correlate to clinical manifestations of indolent systemic mastocytosis. J Allergy Clin Immunol. 2013;132:723-728.

16 Akin C, Valent P, Metcalfe DD. Mast cell activation syndrome: proposed diagnostic criteria. J Allergy Clin Immunol 2010;126:1099-1104.

17 Sotlar K, Escribano L, Landt O et al. One-step detection of c-kit point mutations using peptide nucleic acid-mediated polymerase chain reaction clamping and hybridization probes. Am J Pathol 2003;162: 737-746.

18 Valent $\mathrm{P}$, Escribano L, Broesby-Olsen $\mathrm{S}$ et al. Proposed diagnostic algorithm for patients with suspected mastocytosis: a proposal of the European Competence Network on Mastocytosis. Allergy 2014; 69:1267-1274.

19 Teodosio C, Garcia-Montero AC, Jara-Acevedo et al. An immature immunophenotype of bone marrow mast cells predicts for multilineage D816V KIT mutation in systemic mastocytosis. Leukemia 2012;26:951-958.

20 Matito A, Morgado JM, Alvarez-Twose I et al. Serum tryptase monitoring in indolent systemic mastocytosis: association with disease features and patient outcome. PLoS One 2013;8:e76116.
21 Teodosio C, Garcia-Montero AC, Jara-Acevedo M et al. Gene expression profile of highly purified bone marrow mast cells in systemic mastocytosis. J Allergy Clin Immunol 2013;131:24 e1-24 e4.

22 Alvarez-Twose I, Zanotti R, Gonzalez-de-Olano D et al. Nonaggressive systemic mastocytosis (SM) without skin lesions associated with insect-induced anaphylaxis shows unique features versus other indolent SM. J Allergy Clin Immunol 2014;133:520-528.

23 Butterfield JH, Weiler D, Dewald G. Establishment of an immature mast cell line from a patient with mast cell leukemia. Leuk Res 1988;12:345-355.

24 Pfaffl MW. A new mathematical model for relative quantification in real-time RT-PCR. Nucleic Acids Res 2001;29:e45.

25 Taylor ML, Sehgal D, Raffeld M et al. Demonstration that mast cells, $\mathrm{T}$ cells, and $\mathrm{B}$ cells bearing the activating Kit mutation D816V occur in clusters within the marrow of patients with mastocytosis. J Mol Diagn 2004;6:335-342.

26 Kocabas CN, Yavuz AS, Lipsky PE et al. Analysis of the lineage relationship between mast cells and basophils using the c-kit D816V mutation as a biologic signature. J Allergy Clin Immunol 2005;115:1155-1161.

27 Worobec AS, Semere T, Nagata $\mathrm{H}$ et al. Clinical correlates of the presence of the Asp816Val c-kit mutation in the peripheral blood mononuclear cells of patients with mastocytosis. Cancer 1998;83:2120-2129.

28 Arock M, Sotlar K, Akin C, Broesby-Olsen S et al. Mutation analysis in mast cell neoplasms: recommendations of the European Competence Network on Mastocytosis. Leukemia; advance online publication, 4 February 2015; doi:10.1038/leu.2015.24 (e-pub ahead of print).

29 Kristensen T, Broesby-Olsen S, Vestergaard $\mathrm{H}$ et al. Circulating KIT D816V mutation-positive non-mast cells in peripheral blood are characteristic of indolent systemic mastocytosis. Eur J Haematol 2012;89:42-46.

30 Erben P, Schwaab J, Metzgeroth G et al. The KIT D816V expressed allele burden for diagnosis and disease monitoring of systemic mastocytosis. Ann Hematol 2014;93:81-88.

31 Akin C. Clonality and molecular pathogenesis of mastocytosis. Acta Haematol 2005;114:61-69.

32 Pardanani A, Reeder T, Li CY, Tefferi A. Eosinophils are derived from the neoplastic clone in patients with systemic mastocytosis and eosinophilia. Leuk Res 2003;27:883-885. 